\title{
Effect of Bias Gas Flow on Tracheal Cytokine Concentrations in Ventilated Extremely Preterm Infants: A Randomized Controlled Trial
}

\author{
Katinka P. Bach ${ }^{a}$ Carl A. Kuschel $^{b}$ Nicola Patterson ${ }^{c}$ Hana Skwish $^{d}$

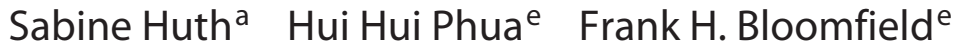

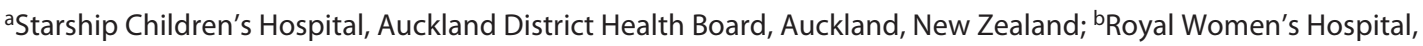 \\ Melbourne, VIC, Australia; 'Middlemore Hospital, Counties Manukau Health, Auckland, New Zealand; \\ dSargent College of Health and Rehabilitation Sciences, Boston University, Boston, MA, USA; ' Liggins Institute, \\ University of Auckland, Auckland, New Zealand
}

\section{Keywords}

Ventilator-induced lung injury · Shear stress · Rheotrauma . Interleukin-8

\begin{abstract}
Background: The objective of this study was to determine whether ventilator bias gas flow affects tracheal aspirate (TA) cytokine concentrations in ventilated extremely preterm infants. Methods: This is a randomized controlled trial in a tertiary neonatal unit in New Zealand. Preterm infants $(<28$ weeks' gestation $/<1,000 \mathrm{~g}$ ) requiring intubation in the first 7 days after birth were randomized to bias gas flows of 4 or 10 $\mathrm{L} / \mathrm{min}$. Cytokine concentrations in TA and plasma were measured at 24,72 , and $120 \mathrm{~h}$ after the onset of ventilation. The primary outcome measure was concentration of interleukin (IL)-8 in TA $24 \mathrm{~h}$ after the onset of mechanical ventilation. Results: Baseline demographics were similar in babies randomized to $4(n=50)$ and $10(n=45) \mathrm{L} / \mathrm{min}$ bias gas flow. TA IL-8 concentrations were not different between groups. Plasma IL-8 concentrations decreased over time $(p<0.05)$. Respiratory support and incidence of bronchopulmonary dysplasia at 36 weeks' corrected gestational age were similar between groups. Fewer babies ventilated at $4 \mathrm{~L} / \mathrm{min}$ developed
\end{abstract}

karger@karger.com www.karger.com/neo

Karger $\stackrel{\text { ' }}{5}$

GOPEN ACCESS
C 2021 The Author(s)

Published by S. Karger AG, Basel

This is an Open Access article licensed under the Creative Commons Attribution-NonCommercial-4.0 International License (CC BY-NC) (http://www.karger.com/Services/OpenAccessLicense), applicable to the online version of the article only. Usage and distribution for commercial purposes requires written permission. necrotizing enterocolitis (NEC) $\geq$ stage 2 ( $n=0$ vs. $n=5 ; p=$ 0.02 ) and fewer died ( $n=1$ vs. $n=5, p=0.06$ ). Conclusions: Lower bias gas flow in ventilated extremely preterm infants did not alter TA cytokine concentrations but the lower incidence of NEC and mortality warrants further investigation.

(c) 2021 The Author(s)

Published by S. Karger AG, Basel

\section{Introduction}

Preterm birth and its consequences remain a major cause of childhood mortality and morbidity. Worldwide, the rate of preterm birth is increasing, with about $1 \%$ of all babies being born before 28 weeks' gestation. Survival rates have improved, but rates of bronchopulmonary dysplasia (BPD) remain high $[1,2]$ and, in many centres, are increasing [3]. BPD in babies born under 28 weeks' gestation in the Australia and New Zealand Neonatal Network, defined as ongoing oxygen or respiratory support at 36 weeks' postmenstrual age, have risen from $44 \%$ in 2010 to $61 \%$ in $2018[4,5]$. BPD is a chronic lung disease charac-

Trial registration number: ACTRN12611000628943.
Correspondence to:

Frank H. Bloomfield, f.bloomfield@auckland.ac.nz 
terized by a disruption of alveolar development with fewer, larger alveoli and decreased vascularity [2]. The longterm health of babies with BPD is affected by more frequent respiratory illnesses, primary healthcare visits, and hospital admissions [2].

$\mathrm{BPD}$ is a multi-factorial disease with ventilator-induced lung injury (VILI) playing an important role. Babies who go on to develop BPD or more severe respiratory distress syndrome (RDS) have been shown to have increased polymorphonuclear leucocytes and macrophages in tracheal aspirates (TAs) from as early as $5 \mathrm{~h}$ of age [6]. These inflammatory cells are accompanied by elevations in pro-inflammatory cytokines, such as interleukin (IL)-8, IL-6, IL-1 $\beta$, and TNFa, which are well-known biomarkers of lung injury $[7,8]$. IL-8, a potent neutrophil chemotactic factor, has been shown to be increased in the first 5 days after birth in TAs of babies who later developed BPD [9] and its elevation precedes the influx of neutrophils in the tracheobronchial effluent [10]. The early nature of the response is supported by correlations of IL- 8 concentrations in amniotic fluid [11] and cord blood [12] with risk of BPD.

Pro-inflammatory mechanisms underlying VILI include volutrauma, barotrauma, atelectrauma, oxytrauma, and biotrauma [2]. This knowledge has led to advances in respiratory care, such as volume-targeted ventilation, which have been demonstrated to reduce death or BPD and number of ventilated days [13]. The role of rheotrau$\mathrm{ma}$, the potential for gas flow to induce injury, has not been well studied in VILI, although the role of shear stress injury in the vascular tree is well described [14]. Most neonatal ventilators apply a continuous or variable bias gas flow in the ventilator circuit, which is used to forcibly inject gas into the lungs during inspiration. Inspiratory flow correlates with bias flow [15], although this relationship is not linear at higher bias flows, presumably due to turbulence generated in the endotracheal tube, which can also lead to a drop in pressure [16]. Studies in term and preterm lambs have demonstrated that high bias gas flows reduce ventilator efficiency [15], increase mRNA expression levels of early response genes, and result in histological changes consistent with VILI after only $2 \mathrm{~h}$ of ventilation [17].

Therefore, we investigated, in ventilated preterm babies, the effect of bias gas flows on inflammatory biomarkers predictive of BPD, with the aim of progressing to a larger RCT with BPD as the primary outcome should the results support this. We hypothesized that low ventilator bias gas flows would result in lower levels of proinflammatory cytokines in TAs, indicative of less severe lung injury.

Bias Flow and Neonatal Lung Inflammation

\section{Methods}

Patients were eligible if born before 28 weeks of gestation or with a birth weight under $1,000 \mathrm{~g}$ and requiring intubation for mechanical ventilation in the first 7 days after birth. Exclusion criteria were cardiorespiratory anomalies, known genetic or chromosomal abnormalities, or expected imminent death. Antenatal consent was sought wherever possible, but ethical approval permitted deferred consent for up to $24 \mathrm{~h}$ when antenatal consent was not possible. Biological samples could only be collected once informed, written consent from parents or guardians was obtained, and this was required for all babies to be included in the trial. The trial protocol was approved by the Multiregion Human Disability and Ethics Committee (MEC/11/08/068) and the hospital Research Review Committee. Babies were randomized at the time the decision to intubate was made to a bias gas flow of 4 or $10 \mathrm{~L} / \mathrm{min}$ using sealed and opaque envelopes. The allocated flow was used each time ventilation was required for the baby up to 36 weeks' postconceptional age and was used for both ventilator (Dräger Babylog 8000plus and VN500) and t-piece resuscitator (Neopuff; Fisher \& Paykel). Ventilator mode was chosen upon discretion of the treating clinician, with volume-targeted mode being the default mode in our unit. Nutrition was provided according to the unit guidelines (https://www.starship.org.nz/guidelines/nutrition-neonatalnutrition-guideline/).

The primary outcome was the concentration of IL-8 in TA at $24 \mathrm{~h}$ after the onset of ventilation. Secondary outcomes were concentration of cytokines IL-1 $\beta$, IL-6, IL-8, IL-10, and TNF $\alpha$ at 24 , 72 , and $120 \mathrm{~h}$ after the onset of ventilation in both TA and blood; respiratory data including number of ventilated days and mode of ventilation; number of days on respiratory support; incidence of $\mathrm{BPD}$, defined as needing supplemental oxygen or respiratory support at 36 weeks' postmenstrual age as collected for the Australia and New Zealand Neonatal Network (ANZNN) registry at the time; and major neonatal morbidities including necrotizing enterocolitis (NEC), defined according to Bell stage 2 or greater. A data safety and monitoring committee was established before the onset of the trial.

TAs were collected over a 12-h period from 24, 72, and $120 \mathrm{~h}$ of onset of ventilation, if still ventilated. Endotracheal suctioning was performed when clinically indicated. Suction catheters were stored at $4^{\circ} \mathrm{C}$ until TA was lavaged into a $1.5-\mathrm{mL}$ Eppendorf tube with $1 \mathrm{~mL} \mathrm{PBS}$, centrifuged for $10 \mathrm{~min}$ at $12,000 \mathrm{~g}$ at $4^{\circ} \mathrm{C}$ with pellet and supernatant stored separately at $-80^{\circ} \mathrm{C}$. Blood samples were collected 24,72 , and $120 \mathrm{~h}$ after the onset of ventilation, centrifuged at $4^{\circ} \mathrm{C}$ for $15 \mathrm{~min}$ at $1,500 \mathrm{~g}$, and stored at $-80^{\circ} \mathrm{C}$. The cytokine concentrations in plasma and TAs were measured using a quantitative magnetic bead-based MILLIPLEX MAP (multianalyte panel) human multiplex cytokine assay (Merck KGaA, Darmstadt, Germany) according to the manufacturer's instructions and blinded to treatment allocation. Plasma samples were analysed undiluted, and TA samples were diluted 3-fold using PBS. As RDS and pulmonary inflammation may alter concentration of both DNA and protein in the airway and there is no accepted standard for normalization of TA samples to account for the potential effect of differing dilutions, no normalization of data was undertaken.

To detect a $35 \%$ reduction in IL- 8 concentration in TA at $24 \mathrm{~h}$ after the onset of ventilation in babies ventilated with a flow of 4 $\mathrm{L} / \mathrm{min}$ compared with babies ventilated with a flow of $10 \mathrm{~L} / \mathrm{min}$ with $80 \%$ power and a 2 -sided significance level of $5 \%$ required a

Neonatology 2021;118:332-339 


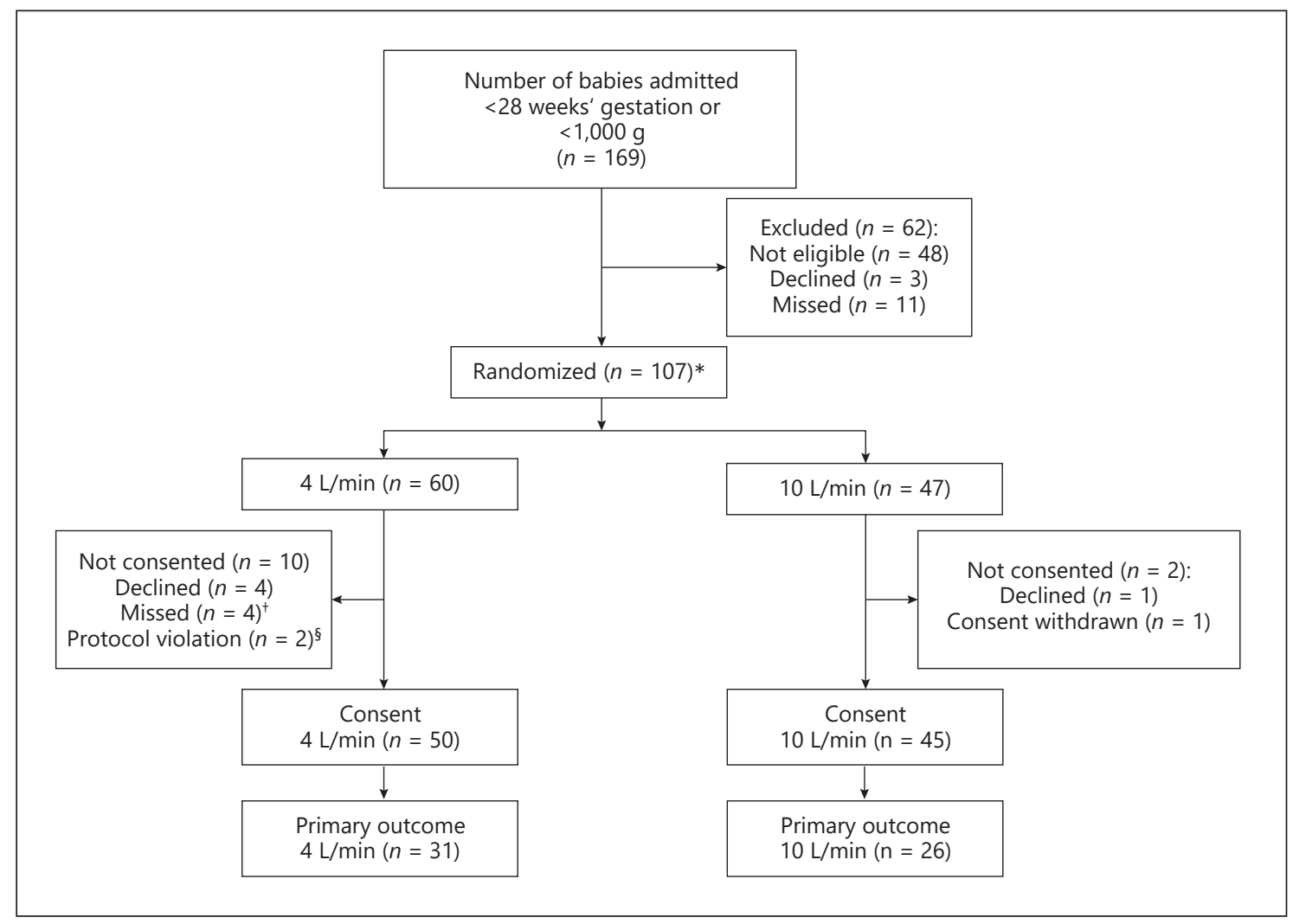

Fig. 1. Consolidated Standards of Reporting Trials diagram for recruitment and treatment assignment. *Antenatal consent was obtained in 18 babies. " Missed" refers to babies who were randomized under the Ethics Committee approval for deferred consent but for whom it was not possible to obtain a decision from the parents/caregivers regarding informed written consent for ongo-

sample size of 128 babies (64 in each arm). Twins or triplets were randomized as individual babies.

Analysis was on an intention-to-treat basis. Non-parametric data were logarithmically transformed to approximate normality, where possible, prior to analysis. The primary outcome and clinical continuous data were analysed using a $t$ test. Categorical data were analysed using $\chi^{2}$ test or Fisher's exact test, where the number in any cell was $<5$, and are presented as relative risk (RR) with $95 \%$ $\mathrm{CI}$. Cytokine concentrations were analysed using a factorial ANO$\mathrm{VA}$ with time as an additional variable.

\section{Results}

One hundred and seven babies were randomized between July 2012 and December 2015, with 95 babies consented (Fig. 1). Due to slower recruitment than anticipated, an interim analysis was performed and the data safety monitoring committee recommended discontinu- ing participation in the trial within the 24 -h time period permitted under the terms of the ethical approval. "Protocol violation": 1 baby received "in and out" surfactant with no ongoing ventilation and 1 baby was in danger of imminent death and died shortly after randomization under deferred consent.

ation of the study on the ground of futility, with little likelihood of demonstrating a difference in the primary outcome. Baseline demographic variables and clinical characteristics were well matched between the 2 groups (Table 1).

The primary outcome of IL- 8 concentration in TA was collected for 31 babies in the $4 \mathrm{~L} / \mathrm{min}$ group and for 26 babies in the $10 \mathrm{~L} / \mathrm{min}$ group; the other babies were extubated prior to $24 \mathrm{~h}$. No significant difference was observed in IL-8 concentration for babies ventilated with 4 versus $10 \mathrm{~L} / \mathrm{min}(16,681[14,351]$ vs. 14,663 [14,816] pg/mL, mean [SD]; Fig. 2a). Concentrations of cytokines IL-1 $\beta$, IL- 6 , IL-8, IL-10, and TNF $\alpha$ were measured at 24, 72, and $120 \mathrm{~h}$ after the onset of ventilation in both TA and blood. No significant differences were found, other than a decrease over time for IL-8 concentration in plasma in both groups $(p<0.01)$ (Fig. 2b). 


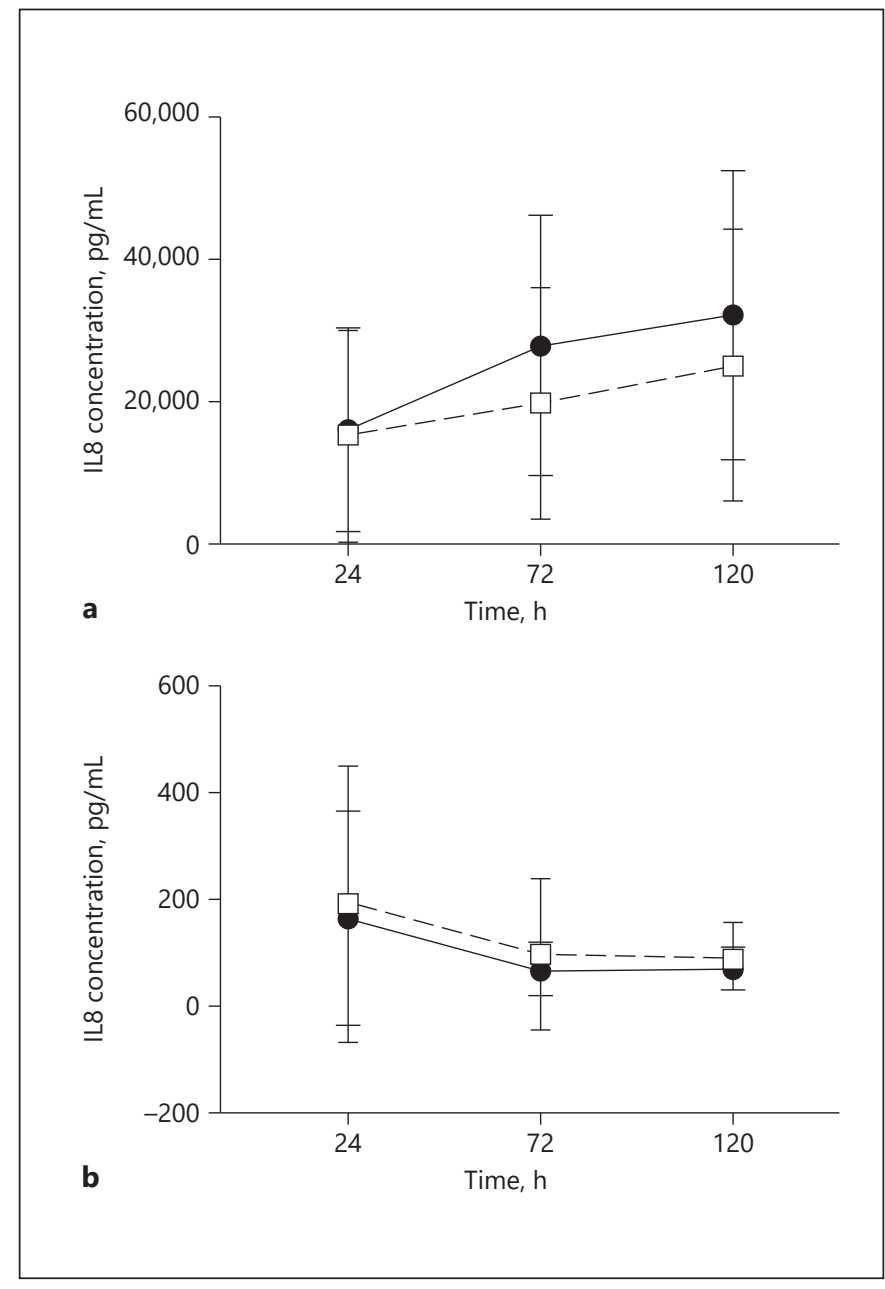

Fig. 2. IL-8 concentration (pg/mL) in TAs (a) and in plasma (b) at 24,72 , and $120 \mathrm{~h}$ after the onset of ventilation. Data are mean with standard deviation. No significant differences were found, other than a decrease over time for IL- 8 concentration in plasma in both groups $(p<0.01)$. $\bullet$, flow $4 \mathrm{~L} / \mathrm{min}$; $\square$, flow $10 \mathrm{~L} / \mathrm{min}$; IL, interleukin; TA, tracheal aspirate.

BPD and the composite outcome for BPD and death were not significantly different between groups, and there was no difference in the median number of ventilated days (Table 2). The bias gas flow did not alter the subsequent number of days on non-invasive ventilation (Table 3). There were no differences in daily maximum peak inspiratory pressure, mean airway pressure, inspiratory time, or fraction of inspired oxygen during mechanical ventilation (data not shown).

Babies ventilated with a flow of $10 \mathrm{~L} / \mathrm{min}$ were more likely to have NEC stage 2 or 3 than babies ventilated with $4 \mathrm{~L} / \mathrm{min}$ ( 5 vs. $0, p=0.02$; Table 4 ), and there also was a trend towards more deaths in the $10 \mathrm{~L} / \mathrm{min}$ group (5 vs.
Table 1. Patient demographics [18]

\begin{tabular}{|c|c|c|}
\hline & $\begin{array}{l}4 \mathrm{~L} / \mathrm{min} \\
(n=48 \\
\text { mothers and } \\
50 \text { babies) }\end{array}$ & $\begin{array}{l}10 \mathrm{~L} / \mathrm{min} \\
(n=38 \\
\text { mothers and } \\
45 \text { babies })\end{array}$ \\
\hline Maternal age, years & $30.8(6.4)$ & $29.9(6.9)$ \\
\hline \multicolumn{3}{|l|}{ Maternal ethnicity } \\
\hline European & $19(40)$ & $10(26)$ \\
\hline Maori & $7(15)$ & $12(32)$ \\
\hline Asian & $14(29)$ & $10(26)$ \\
\hline Pacific & $7(15)$ & $4(11)$ \\
\hline Other & $1(2)$ & $2(5)$ \\
\hline Caesarean section & $26(54)$ & $21(55)$ \\
\hline Preterm labour & $31(65)$ & $26(68)$ \\
\hline Preterm rupture of membranes & $23(48)$ & $18(47)$ \\
\hline \multicolumn{3}{|l|}{ Chorioamnionitis } \\
\hline Clinical & $21(44)$ & $20(63)$ \\
\hline Histological & $28 / 50(56)$ & $20 / 45(44)$ \\
\hline Any antenatal steroids & $47(98)$ & $37(97)$ \\
\hline$<24 \mathrm{~h}$ & $10(21)$ & $13(34)$ \\
\hline 1-7 days & $30(63)$ & $18(47)$ \\
\hline$>7$ days & $6(13)$ & $3(8)$ \\
\hline Multiple courses & $1(2)$ & $3(8)$ \\
\hline None & $1(2)$ & $1(3)$ \\
\hline Gestation, weeks & $25.9(1.4)$ & $25.4(1.3)$ \\
\hline Birth weight, $g$ & $813(167)$ & $828(152)$ \\
\hline SGA & $5(10)$ & $3(7)$ \\
\hline Male sex & $29(57)$ & $19(42)$ \\
\hline Singleton & $40(80)$ & $28(62)$ \\
\hline One of twins & $10(20)$ & $14(31)$ \\
\hline One of triplets & 0 & $3(7)$ \\
\hline APGAR $1 \mathrm{~min}$ & $6(1-9)$ & $5(1-9)$ \\
\hline APGAR $5 \mathrm{~min}$ & $7(3-10)$ & $7(2-9)$ \\
\hline
\end{tabular}

Data are presented as mean (SD); number/total number of babies or mothers, $n$ (\%); or median (range), as appropriate. SGA is defined as birth weight below the 10th percentile [18]. SGA, small for gestational age.

$1, p=0.06$; Table 4). Of the 5 babies who developed NEC, 3 required surgery following which 2 died. Two further babies died beyond term-equivalent age from severe BPD, 1 additionally complicated with neurofibromatosis type 1. Other secondary clinical outcomes were not significantly different between groups (Table 4).

\section{Discussion}

Contrary to our hypothesis, we found no effect of bias gas flow on concentrations of inflammatory cytokines that have been associated with later risk of BPD. Al- 
Table 2. Cytokine concentrations in TA and plasma

\begin{tabular}{|c|c|c|c|c|c|c|}
\hline \multirow[t]{2}{*}{ Time, $\mathrm{h}$} & \multicolumn{3}{|l|}{$4 \mathrm{~L} / \min (n=50)$} & \multicolumn{3}{|l|}{$10 \mathrm{~L} / \min (n=45)$} \\
\hline & 24 & 72 & 120 & 24 & 72 & 120 \\
\hline \multicolumn{7}{|l|}{$T A, p g / m L$} \\
\hline IL $-1 \beta$ & $143(1.58-5,225)$ & $567(1.10-17,949)$ & $2,171(23.6-16,998)$ & $61.4(2.19-2,282)$ & $369(4.56-6,745)$ & $4,040(4.41-43,570)$ \\
\hline IL-6 & $1,510(2.7-9,559)$ & $1,233(57.5-10,469)$ & $2,686(95.8-30,000)$ & $635(5.38-5,486)$ & $705(36.6-35,288)$ & $2,312(97.6-33,845)$ \\
\hline TNFa & $68.8(3.17-2,225)$ & $159(1.07-1,564)$ & $467(23.4-5,945)$ & $56.3(2.06-828)$ & $173(8.77-3,136)$ & $514(6.56-10,475)$ \\
\hline IL-10 & $31.3(3.3-3,510)$ & $43.8(1.84-1,168)$ & $125(17.1-1,089)$ & $45.9(2.82-546)$ & $52.7(3.30-2,949)$ & $172(6.91-3,694)$ \\
\hline \multicolumn{7}{|l|}{ Plasma, $p g / m L$} \\
\hline IL-1 $\beta$ & $0.80(0.02-2.84)$ & $0.80(0.02-0.80)$ & $0.80(0.01-5.19)$ & $0.78(0.02-7.48)$ & $0.80(0.02-2.49)$ & $0.65(0.02-1.00)$ \\
\hline IL-6 & $38.3(0.71-1,035)$ & $9.73(0.90-114)$ & $32.4(0.90-191)$ & $23.6(0.90-550)$ & $15.4(0.85-327)$ & $26.5(1.02-399)$ \\
\hline $\mathrm{TNF} \alpha$ & $23.4(7.42-47.3)$ & $15.2(2.58-36.0)$ & $17.5(0.70-55.9)$ & $22.0(2.68-52.2)$ & $20.5(3.13-45.7)$ & $20.0(3.21-83.1)$ \\
\hline IL-10 & $22.0(1.02-329))$ & $7.08(0.38-136)$ & $6.72(2.09-55.3)$ & $17.5(1.02-123)$ & $7.86(0.53-45.6)$ & $10.0(2.19-527)$ \\
\hline
\end{tabular}

Data are presented as median (range). There were no statistically significant differences between groups. TA, tracheal aspirate; IL, interleukin.

Table 3. Secondary respiratory outcomes

\begin{tabular}{lccc}
\hline & $4 \mathrm{~L} / \min (n=50)$ & $10 \mathrm{~L} / \min (n=45)$ & $p$ value \\
\hline Respiratory support, days & $7(1-76)$ & $8(1-43)$ & 0.66 \\
CV & $0(0-17)$ & $0(0-26)$ & 0.52 \\
HFO & $37.5(1-61)$ & $40(0-66)$ & 0.72 \\
CPAP & $11(0-42)$ & $10(0-43)$ & 0.95 \\
HF & $0(0-8)$ & $0(0-6)$ & 0.76 \\
LF & & RR $(95 \% \mathrm{CI})$ \\
\hline BPD & $42(84)$ & $35(77.8)$ & $1.08(0.89-1.32)$ \\
\hline BPD & $42(84)$ & $37(82.2)$ & $1.02(0.85-1.23)$ \\
BPD or death & & & \\
\hline
\end{tabular}

Data are presented as median (range) or $n(\%)$. CV, conventional ventilation; HFO, high-frequency oscillatory ventilation; CPAP, continuous positive airway pressure; HF, high flow; LF, low flow; RR, relative risk; BPD, bronchopulmonary dysplasia.

Table 4. Secondary clinical outcomes

\begin{tabular}{lccc}
\hline & $4 \mathrm{~L} / \min (n=50)$ & $10 \mathrm{~L} / \min (n=45)$ & $\mathrm{RR}(95 \% \mathrm{CI})$ \\
\hline Death & $1(2)$ & $5(11.1)$ & $0.18(0.02-1.48)$ \\
Infection & $1^{\#}$ & $1^{\S}$ & \\
NEC & & 2 & \\
BPD & 0 & $5(11.1)^{\wedge}$ & Not estimable \\
NEC (stages 2-3) & $1(2)$ & $3(6.7)$ & $0.30(0.03-2.78)$ \\
IVH (grades 3-4) & $4(8)$ & $4(8.9)$ & $0.90(0.24-3.39)$ \\
PVL & $5(10)$ & $3(6.7)$ & $1.47(0.37-5.79)$ \\
ROP (zgrade 3 or plus disease) & $18(36)$ & $20(44.4)$ & $0.81(0.49-1.33)$ \\
PDA (received treatment) & $2(4)$ & $3(6.7)$ & $0.60(0.10-3.43)$ \\
Pulmonary haemorrhage & & &
\end{tabular}

RR, relative risk; BPD, bronchopulmonary dysplasia; NEC, necrotizing enterocolitis (Bell staging); IVH, intraventricular haemorrhage (Papile classification); ROP, retinopathy of prematurity; PDA, patent ductus arteriosus. ${ }^{\#}$ Infection - culture positive. ${ }^{\S}$ Infection - culture negative. ${ }^{\wedge}$ Delivered at 24 and 25 weeks' gestation, death at 2 months' corrected gestational age ( 1 following complicated respiratory cause post-oesophageal perforation, 1 additionally diagnosed with neurofibromatosis type I). ${ }^{*} p<0.05$. 
though the trial was stopped early after an interim analysis was conducted due to slow recruitment, the lack of any difference indicates that this finding was unlikely to change even if the sample size had been reached. A key factor for slow recruitment was the introduction of variable bias flow ventilators in units that initially had expressed interest in participating, preventing their participation and leaving recruitment to a single centre. Although the increasingly widespread use of variable flow ventilators in neonatal intensive care may be considered to render the hypothesis of rheotrauma redundant, the flows generated by these ventilators can be substantially higher than the $10 \mathrm{~L} / \mathrm{min}$ in the higher flow group in this study, yet clinicians may not even be aware of the flows generated. The primary outcome of a biological variable at $24 \mathrm{~h}$ means that this is unlikely to have been affected by any potential bias from an unblinded trial, including that ventilatory strategy and time of extubation were not protocolized but performed according to the unit guidelines with the final decision left to the clinician. However, extubation prior to $24 \mathrm{~h}$ occurred in a significant number of babies, meaning that samples for the primary outcome were only available in approximately $2 / 3$ of babies enrolled.

The concept of shear stress is widely accepted in the pathology of cardiovascular disorders such as atherosclerosis [14]. Indeed, forces generated by flow are considered important in driving developmental processes in several organ systems, including the cardiovascular tree [19]. BPD increasingly is recognized as a disease of disrupted development [2], rather than simply destructive lung injury, and it is possible that flow, whether of fetal lung fluid or of air postnatally when the lung is still developing, also contributes to development of the lung.

Experiments comparing 3 different bias gas flows during ventilation of preterm lambs demonstrated lower ventilatory efficiency; upregulation of early response genes, known to correlate with lung injury, in pulmonary tissue; and greater histological lung injury in the animals ventilated at higher compared with the lowest flows, even after only $2 \mathrm{~h}$ of mechanical ventilation using ventilatory management similar to those used in neonatal nurseries $[15,17]$. In vitro experiments using models of human neonates confirm potentially injurious jets of air impinging upon the carina that are modulated by the ventilatory strategy employed [20]. Animal and in vitro experiments can be carried out in a highly controlled setting with direct analysis of effects on tissue, whereas this is not feasible in a human trial.

Bias Flow and Neonatal Lung Inflammation
There are a multitude of maternal and perinatal factors that can potentially influence cytokine release, evident by reports finding associations between later risk of BPD and cytokine concentrations in amniotic fluid [11] and cord blood [12], clearly representing factors operating prior to birth, that may mask any potential effect of bias gas flow from mechanical ventilation. Plasma cytokine concentrations were 1-2 orders of magnitude less than TA concentrations, consistent with previous reports in infants and adults $[21,22]$, and did not follow the same general trend of an increase over time. This indicates that plasma cytokine concentrations may not be a reliable marker of pulmonary inflammation in the preterm baby. We are not aware of other paired TA and plasma analyses over time in neonates, and this requires confirmation in a larger sample.

Although approval for deferred consent meant we were able to commence the allocated flow with the onset of mechanical ventilation, we could not obtain biological samples until informed, written consent had been obtained, and to ensure sufficient time for this to happen in families for whom antenatal consent was not possible, the first sample was collected at $24 \mathrm{~h}$. It is, therefore, possible that we may have missed an early rise in TA cytokine concentrations as inflammatory cytokines may rise within the first hour of injurious ventilation [23]. It is also possible that we may have missed a later effect of bias flow on pulmonary cytokine concentrations as the last TA was taken at $120 \mathrm{~h}$. Future studies could consider both later samples and also measurement of a wider range of antiinflammatory cytokines.

We defined BPD as needing supplemental oxygen or respiratory support at 36 weeks, as this was the definition in use by the ANZNN at that time; since then, the AN$\mathrm{ZNN}$ registry records physiological $\mathrm{BPD}[24]$ as a more objective assessment. However, it is unlikely that use of the physiological definition of BPD would change our findings. Babies in this trial were ventilated for longer and had a higher incidence of BPD compared with all infants born at less than 28 weeks' gestation or less than $1,000 \mathrm{~g}$ at birth [25], reflecting a select group of high-risk babies requiring ventilation in the first week after birth in an era when non-invasive ventilatory support increasingly is the mode of choice.

An unexpected outcome for this study was the higher incidence of stage 2 or 3 NEC, and a trend towards greater mortality, in babies ventilated with higher bias gas flow. Caution is warranted in interpreting these data, as this may be a type I error, as the number of cases is low and 2 of the deaths in the higher flow group 
were from multi-morbid complications of extremely preterm birth.

This is the first neonatal study of bias gas flow in the clinical setting; however, there are several limitations including single centre recruitment, which was halted at $75 \%$ of the planned sample size. Nevertheless, newer generations of neonatal ventilators apply a slope function or variable inspiratory flow to reach inspiratory pressure within a clinician-determined time limit. This requires the bias flow to be variable and frequently much greater than $10 \mathrm{~L} / \mathrm{min}$. Given our findings, further research into potential adverse effects of high bias gas flows may be warranted.

\section{Conclusion}

Reduced bias gas flow did not affect early pro-inflammatory TA cytokine responses in ventilated ELBW infants. The finding of decreased NEC is limited by a small sample size and low incidence of NEC. The much higher bias gas flows reached in modern variable flow ventilators, and whether there is any association with NEC, may merit further investigation. This could initially be performed using in vitro or in silico approaches, as the sample size for studies attempting to reduce the incidence of $\mathrm{BPD}$ is very large.

\section{Statement of Ethics}

The research described in this article was conducted ethically in accordance with the World Medical Association Declaration of Helsinki. The trial was approved by the Multiregion Human Disability and Ethics Committee (MEC/11/08/068), and the hospital's Research Review Committee. Informed written consent was obtained for all participants from the parents/caregivers. The trial registration number is Australian New Zealand Clinical Trials Registry Number ACTRN12611000628943.

\section{Conflict of Interest Statement}

The authors have no disclosures to make.

\section{Funding Sources}

This work was supported by a Clinical Training Fellowship grant from the Health Research Council of New Zealand (to K.P.B, No. 14/597), a Cure Kids project grant (No. 3548), and a philanthropic donation.

\section{Author Contributions}

K.P.B., C.A.K., and F.H.B. designed the study, developed the protocols, and oversaw the trial and collection of data. N.P., H.S., S.H., and H.H.P. contributed substantially to acquisition and analysis of data. K.P.B. and F.H.B. drafted the manuscript, which was reviewed and contributed to by all authors.

\section{References}

1 Siffel C, Kistler KD, Lewis JFM, Sarda SP. Global incidence of bronchopulmonary dysplasia among extremely preterm infants: a systematic literature review. J Matern Fetal Neonatal Med. 2019 Aug 9:1-11.

2 Thebaud B, Goss KN, Laughon M, Whitsett JA, Abman SH, Steinhorn RH, et al. Bronchopulmonary dysplasia. Nat Rev Dis Primers. 2019 Nov 14;5(1):78.

3 Jensen EA. Prevention of bronchopulmonary dysplasia: a summary of evidence-based strategies. Neoreviews. 2019 Apr;20(4):e189-201.

4 Chow SSW. Report of the Australian and New Zealand neonatal network 2010. Sydney: ANZNN; 2013.

5 Chow SSW, Creighton P, Chambers GM, Lui K. Report of the Australian and New Zealand neonatal network 2018. Sydney: ANZNN; 2020.

6 Murch SH, Costeloe K, Klein NJ, MacDonald TT. Early production of macrophage inflammatory protein- 1 alpha occurs in respiratory distress syndrome and is associated with poor outcome. Pediatr Res. 1996 Sept;40(3):490-7.

7 Lista G, Castoldi F, Fontana P, Reali R, Reggiani A, Bianchi S, et al. Lung inflammation in preterm infants with respiratory distress syndrome: effects of ventilation with different tidal volumes. Pediatr Pulmonol. 2006 Apr; 41(4):357-63.

8 Speer CP. Pulmonary inflammation and bronchopulmonary dysplasia. J Perinatol. 2006;26(Suppl 1):S57-4.

9 Huang HC, Tai FY, Wang FS, Liu CA, Hsu TY, Ou CY, et al. Correlation of augmented IL-8 production to premature chronic lung disease: implication of posttranscriptional regulation. Pediatr Res. 2005 Aug;58(2):21621.

10 Munshi UK, Niu JO, Siddiq MM, Parton LA. Elevation of interleukin-8 and interleukin- 6 precedes the influx of neutrophils in tracheal aspirates from preterm infants who develop bronchopulmonary dysplasia. Pediatr Pulmonol. 1997 Nov;24(5):331-6.

11 Yoon BH, Romero R, Jun JK, Park KH, Park JD, Ghezzi F, et al. Amniotic fluid cytokines (interleukin-6, tumor necrosis factor-alpha, interleukin-1 beta, and interleukin-8) and the risk for the development of bronchopulmonary dysplasia. Am J Obstet Gynecol. 1997 Oct;177(4):825-30.
12 Rocha G, Proença E, Guedes A, Carvalho C, Areias A, Ramos JP, et al. Cord blood levels of IL-6, IL-8 and IL-10 may be early predictors of bronchopulmonary dysplasia in preterm newborns small for gestational age. Dis Markers. 2012;33(1):51-60.

13 Klingenberg C, Wheeler KI, McCallion N, Morley CJ, Davis PG. Volume-targeted versus pressure-limited ventilation in neonates. Cochrane Database Syst Rev. 2017 Oct 17;10: CD003666

14 Cameron JN, Mehta OH, Michail M, Chan J, Nicholls SJ, Bennett MR, et al. Exploring the relationship between biomechanical stresses and coronary atherosclerosis. Atherosclerosis. 2020 Jun; 302:43-51.

15 Bach KP, Kuschel CA, Oliver MH, Bloomfield FH. Ventilator gas flow rates affect inspiratory time and ventilator efficiency index in term lambs. Neonatology. 2009;96(4):25964.

16 Jarreau PH, Louis B, Dassieu G, Desfrere L, Blanchard PW, Moriette G, et al. Estimation of inspiratory pressure drop in neonatal and pediatric endotracheal tubes. J Appl Physiol. 1999 Jul;87(1):36-46. 
17 Bach KP, Kuschel CA, Hooper SB, Bertram J, McKnight S, Peachey SE, et al. High bias gas flows increase lung injury in the ventilated preterm lamb. PLoS One. 2012;7(10):e47044.

18 Fenton TR, Kim JH. A systematic review and meta-analysis to revise the Fenton growth chart for preterm infants. BMC Pediatr. 2013; 13:59.

19 Daems M, Peacock HM, Jones EAV. Fluid flow as a driver of embryonic morphogenesis. Development. 2020 Aug 7;147(15):147.

20 Nof E, Heller-Algazi M, Coletti F, Waisman D, Sznitman J. Ventilation-induced jet suggests biotrauma in reconstructed airways of the intubated neonate. J R Soc Interface. 2020 Jan;17(162):20190516.
21 Wrigge $\mathrm{H}$, Uhlig U, Zinserling J, BehrendsCallsen E, Ottersbach G, Fischer M, et al. The effects of different ventilatory settings on pulmonary and systemic inflammatory responses during major surgery. Anesth Analg. 2004 Mar;98(3):775-contents.

22 Harris MC, D'Angio CT, Gallagher PR, Kaufman D, Evans J, Kilpatrick L. Cytokine elaboration in critically ill infants with bacterial sepsis, necrotizing entercolitis, or sepsis syndrome: correlation with clinical parameters of inflammation and mortality. J Pediatr. 2005 Oct;147(4):462-8.
23 Chiumello D, Pristine G, Slutsky AS. Mechanical ventilation affects local and systemic cytokines in an animal model of acute respiratory distress syndrome. Am J Respir Crit Care Med. 1999 Jul;160(1):109-16.

24 Walsh MC, Wilson-Costello D, Zadell A, Newman N, Fanaroff A. Safety, reliability, and validity of a physiologic definition of bronchopulmonary dysplasia. J Perinatol. 2003 Sept;23(6):451-6

25 Board ADH. National women's annual clinical report 2017. Auckland, New Zealand: Auckland District Health Board; 2017. 